EXTENDED REPORT

\title{
The risk of complications of uveitis in a district hospital cohort
}

\author{
R Maini, J O'Sullivan, A Reddy, S Watson, C Edelsten
}

Br J Ophthalmol 2004;88:512-517. doi: 10.1136/bjo.2002.013334

See end of article for authors' affiliations .....................

Correspondence to: Clive Edelsten, Ophthalmology Department, Ipswich Hospital, Ipswich IP4 5PD, UK; edelsten@easynet. co.uk

Accepted for publication 28 May 2003

\begin{abstract}
Aims: To establish the frequency and risk factors for visual loss in a primary referral cohort of hospital patients with uveitis.

Methods: 561 consecutive uveitis patients attending three district hospitals were recruited and the acuity at the end of the study period recorded. A retrospective case-control study of risk factors for visual loss (permanent loss of acuity $<6 / 9$ ) was performed. Risk factors examined included type of uveitis, age at onset of uveitis, race, type of systemic inflammatory disease, length of follow up, and treatment variables. Results: Visual loss of at least 6/12 in one eye was found in 111 patients (19.9\%). Only four patients $(0.7 \%$ ) suffered severe bilateral visual loss (6/36 or less). Visual loss was associated with age at onset $>60$ years (odds ratio 3.9, 95\% confidence interval (Cl) 2.2 to 7.0 , long follow up 2.0 (1.2 to 3.3) and a history of cataract surgery $3.9(2.1$ to 7.2$)$. It was less likely in patients with acute anterior uveitis $0.2(0.1$ to 0.3$)$. Conclusion: The frequency of visual loss associated with uveitis in a district hospital cohort is less than that found in referral centres and levels of legal blindness are low. Although acute anterior uveitis has a low frequency of visual loss it contributes significantly to the total burden. The ocular co-morbidity of the elderly may contribute to the increased visual loss of late onset uveitis.
\end{abstract}

$\mathrm{T}$ he main sight threatening complications of uveitis are cataract formation, glaucoma, and maculopathy; these complications also have an increasing prevalence in the normal elderly population. ${ }^{1}$ The aetiologies of uveitis change with age and children aged less than 10 years have a very different range of diagnoses and complications compared to the adult uveitis population. ${ }^{2}{ }^{3}$ There is little information on the relation of increasing age of onset to the prevalence of complications in the adult uveitis population apart from a reported decrease in prevalence of intermediate uveitis and an increase in secondary ocular hypertension. ${ }^{4}$ Recent population based studies have demonstrated that glaucoma, cataract, and macular degeneration are the major causes of visual impairment in industrial societies and their prevalence increases markedly with age. ${ }^{56}$ Uveitis is thought to cause a significant level of blindness in the working population yet is not mentioned as a cause of bilateral visual loss in either study and only one case of monocular blindness ascribed to uveitis was found in 3821 subjects aged more than 65 years. Population based surveys of younger subjects will require very large numbers if meaningful estimates of the prevalence of visual loss from uveitis are to be obtained. A population based study from India found a prevalence of $0.73 \%$ for active or inactive uveitis unrelated to previous trauma or surgery. ${ }^{7}$ The rate of visual loss was not reported. This study may have underestimated the prevalence of uveitis since cases that did not leave signs of irreversible damage were not recorded.

Few patients with uveitis now develop severe visual loss warranting blind registration. The great majority of uveitis patients with visual loss have mild bilateral or unilateral impairment and therefore only limited epidemiological data can be gained from national blind registration statistics. Regional studies of uveitis are biased by geographical and racial differences in the aetiology of uveitis and the referral patterns of uveitis patients differ widely between medical systems. In the United Kingdom, hospital based studies are likely to provide a relatively complete population of symptomatic uveitis: general practitioners rarely treat any condition requiring topical steroids and refer patients directly to hospital eye departments and the referral of asymptomatic uveitis from routine optometric examination is infrequent in adults.

There are few studies documenting the frequency of visual loss in the general uveitis population. In a hospital based study from Minnesota from 1962, Darrell $e t$ al ${ }^{8}$ found a rate of visual loss (defined as $<25 \%$ of vision) in patients with uveitis of $6 \%$ and Rothova found a similar rate of visual loss $(<6 / 60)$ in a mixed primary and secondary referral centre over 30 years later. ${ }^{1}$ The latter study found cystoid macular oedema, corneal opacities, and macular inflammatory lesions to be the major causes of visual loss resulting from intraocular inflammation. To our knowledge there have been no other large studies of the complication rate of the primary referral uveitis population.

The current study was performed to establish the frequency of visual loss and other significant complications such as ocular hypertension and the administration of systemic treatment or surgery in a large series of uveitis patients from three English primary referral centres. The risk factors for visual loss were examined. As the major complications of uveitis are cataract, glaucoma, and maculopathy and these have a markedly increased prevalence in the normal elderly population, we specifically examined the influence of age at onset on the complication rate in uveitis patients. Establishing the frequency and causes of visual loss in the primary uveitis population will aid the design of specialist referral services.

\section{METHODS}

In all, 561 consecutively presenting adult patients with uveitis were assessed at three large district general hospitals (Ipswich Hospital, Suffolk; Frimley Park Hospital and the Prince Charles Eye Unit, Windsor). New and longstanding patients were recruited for 3 years at Ipswich, 1 year at Windsor, and 6 months at Frimley Park. At the end of the study period the level of permanent visual impairment was recorded in those under hospital follow up, or the acuity at the final visit of those discharged from regular follow up. We 
excluded all patients presenting with their first episode of uveitis under the age of 10 years and patients referred from outside the normal hospital catchment areas for specialist opinion.

A retrospective case-control study analysed the frequency of visual loss and other complications and the risk factors for visual loss were established. Baseline variables noted included age at onset of uveitis, race (white or not), and sex. Age and length of follow up at the time of final assessment were also recorded.

Uveitis was categorised into five clinical types: (1) acute painful anterior, (2) chronic painless anterior with iris changes of heterochromia or transillumination, (3) other chronic anterior, (4) posterior uveitis with choroidal involvement, (5) other posterior uveitis. The anatomical classification proposed by the International Uveitis Study Group ${ }^{9}$ was not used, as it was felt necessary to use a classification with a greater correlation with aetiology and outcome. In populations of north European origin the majority of patients have acute painful anterior uveitis and this type has a markedly different outcome and association with systemic disease from other forms of anterior uveitis which are grouped together in the IUSG scheme. ${ }^{10}$ Choroiditis has been found to be a major risk factor for visual loss in posterior segment inflammation, ${ }^{1}$ but it may be classified in both posterior and panuveitis in the IUSG scheme. Therefore, our classification separated these two categories as well as the types of anterior uveitis known to be associated with high rates of cataract and secondary ocular hypertension. ${ }^{11-13}$ Chronic painless anterior uveitis with iris changes (type 2) includes both cases of Fuchs' heterochromic cyclitis and those of presumed herpetic aetiology in the absence of previous keratitis. ${ }^{14}$ Herpes simplex virus uveitis has been shown to produce raised intraocular pressure in $90 \%$ of cases. ${ }^{15}$

For the purposes of statistical analysis systemic diseases associated with uveitis were grouped into three categories: group 1, no known systemic disease associated with uveitis; group 2, ankylosing spondylitis, inflammatory bowel disease, psoriasis, or diabetes mellitus; and group 3, sarcoidosis, multiple sclerosis, or Behçet's disease. Diseases in group 2 are most commonly associated with anterior uveitis and those in group 3 are most commonly associated with posterior segment disease.

The main outcome variable was visual acuity, defined as the best visual acuity recorded in the 6 months before the acuity at the time of final assessment. Visual acuity in patients with uveitis varies reversibly with disease activity as well as in those developing surgical complications. In order to exclude patients with temporary visual loss at the time of their final assessment, the best acuity in the previous 6 months of those with varying acuity was used. The visual acuity of patients with cataract awaiting surgery at the time of final assessment was assigned the acuity following cataract extraction.

Visual acuity was stratified into five levels defined by the corrected Snellen acuity of the worse eye: $6 / 9$ or better in both eyes $=$ level $1 ; 6 / 9$ or better in better eye and 6/12-6/24 worse eye $=$ level $2 ; 6 / 9$ or better in better eye and $6 / 36$ or worse in the worse eye $=$ level $3 ; 6 / 12-6 / 24$ in the better eye $=$ level $4 ; 6 / 36$ or worse in the better eye $=$ level 5 . A binary assessment of visual loss was also used-any eye less than $6 / 9$. Other variables recorded included treatment and disease severity factors: cataract extraction, trabeculectomy, the administration of periocular or systemic immunosuppression, and secondary ocular hypertension defined as an intraocular pressure more than $24 \mathrm{~mm} \mathrm{Hg}$ recorded on more than one occasion.

\section{Statistical analysis}

The association between baseline and other variables with any visual loss was established by univariate analysis. Age and follow up time were treated as binary variables for the multivariate analyses. Significant variables were entered into a multivariate logistic regression analysis controlled for referral centre. The initial model only included baseline variables; there was no significant difference in the significance of the baseline variables when the treatment variables were included and only the results of the full model are presented. The analyses were also repeated in the subgroups of acute anterior uveitis (type 1) and all other types of uveitis (types 2-5). Odds ratios are presented with 95\% confidence intervals. All analyses were performed with Stata version 6.

\section{RESULTS}

A total of 561 consecutive patients were included in the study. The demographic details are shown in table 1. The mean age at onset was 43.1 years and the median follow up was 4 years. The modal decade of onset was the fourth (fig l). Patients with posterior uveitis and no choroiditis (type 5) had the youngest mean age of onset at 40 years and those with chronic anterior uveitis associated with iris changes (type 2) had the oldest at 48 years (table 1).

The most common type of uveitis was acute anterior uveitis (type 1) 345/561 (61\%), followed by posterior uveitis without choroidal involvement (type 5), 103/561 (18\%) In 325 (58\%) patients there was no associated systemic disease. Acute anterior uveitis and posterior uveitis had the highest frequency of systemic disease and choroiditis the lowest (table 2) group 2 diseases were most frequent in those with acute anterior uveitis 155/344 (45\%) and group 3 diseases were found most frequently in those with posterior uveitis without choroidal involvement (25\%).

Eighty three (14.8\%) required systemic treatment; 88 ( $15.7 \%$ ) had secondary ocular hypertension (table 3 ). Eighty nine patients (15.9\%) underwent a surgical procedure including $67(11.9 \%)$ with cataract extraction and 13 $(2.3 \%)$ with trabeculectomy. Other procedures included laser iridotomy, vitrectomy, and detachment surgery.

Chronic anterior uveitis with iris changes (type 2) had a higher frequency of all complications than any other type of uveitis apart from the need for systemic treatment. Acute

\begin{tabular}{llllll} 
Table 1 & Demographic details of patients & & \\
\hline Uveitis type & Total & $\begin{array}{l}\text { Age (SD) (range) } \\
\text { at onset }\end{array}$ & $\begin{array}{l}\text { Age (SD) (range) } \\
\text { at assessment }\end{array}$ & Non-white & Male \\
\hline Acute anterior & 344 & $42(16)(10-89)$ & $48(16)(12-91)$ & $18(5 \%)$ & $171(50 \%)$ \\
Chronic anterior (iris) & 27 & $43(17)(12-75)$ & $54(17)(24-80)$ & 0 & $18(67 \%)$ \\
Chronic anterior & 45 & $47(18)(14-82)$ & $53(18)(15-84)$ & $3(7 \%)$ & $24(53 \%)$ \\
Choroiditis & 44 & $41(19)(11-77)$ & $52(19)(16-80)$ & $2(5 \%)$ & $15(34 \%)$ \\
Posterior uveitis & 101 & $40(18)(10-89)$ & $49(19)(13-90)$ & $10(10 \%)$ & $52(51 \%)$ \\
\hline
\end{tabular}




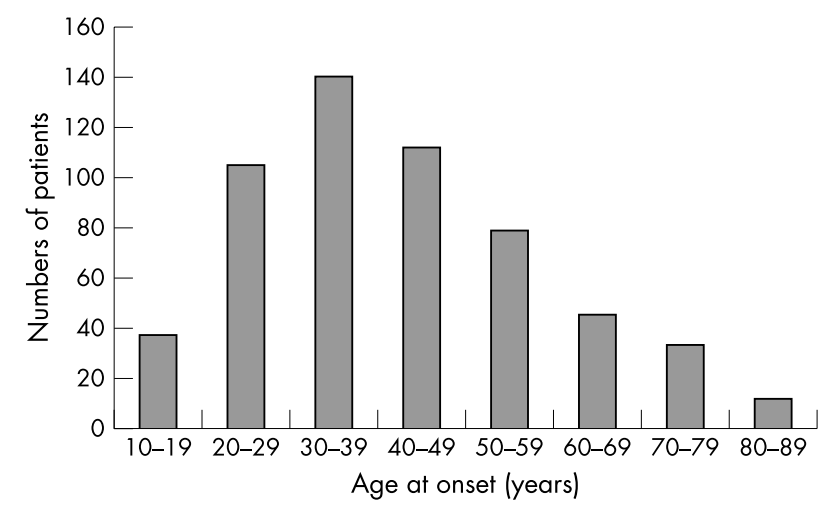

Figure 1 Age at onset of uveitis.

anterior uveitis was associated with the lowest rate of complications apart from the use of systemic treatment and the frequency of trabeculectomy. The highest rate of systemic treatment was found in choroiditis and the lowest rate was found in chronic anterior uveitis without iris changes (type 3).

There were 111 (19.9\%) patients with visual loss (table 4$)$. These included 54 (9.6\%) with mild unilateral loss; 32 (5.7\%) with severe unilateral; 20 (3.6\%) with mild bilateral; and four $(0.7 \%)$ with severe bilateral visual loss.

The largest numbers of patients with any visual loss were found equally in those with acute anterior uveitis (31 patients) and posterior uveitis without choroiditis. ${ }^{30}$ Posterior uveitis without choroiditis had the greatest number of patients with bilateral visual loss (eight patients). The highest frequency of visual loss was found in those with chronic anterior uveitis with iris changes (48\%).
Older age at the onset of uveitis ( $>60$ years) was significantly associated with female sex, ocular hypertension, surgery, and visual loss but a shorter follow up period (table 5).

Visual loss was independently associated with the baseline variables of non-white race, hospital site, uveitis other than acute anterior uveitis (types 2-5), older age at onset of uveitis, and longer follow up (table 6). There was a weak association with the presence of systemic disease: $14 \%$ of those with visual loss had group 2 diseases compared to $7 \%$ of those with no visual loss. All treatment variables were associated with an increased risk of visual loss, including cataract extraction, trabeculectomy, and systemic treatment.

On multivariate analysis, using any visual loss as the outcome variable, the following variables were independently associated with visual loss: age at onset of more than 60 years, uveitis types $2-5$, follow up of more than 4 years, cataract surgery, and hospital (table 7). In a subgroup analysis by type of uveitis age at onset of uveitis, systemic treatment, and cataract surgery were again significantly associated with visual loss. Patients with acute anterior uveitis had no other risk factors for visual loss. In patients with other forms of uveitis, non- white race and long follow up were additional risk factors.

When patients were stratified by the main baseline risk factors of type of uveitis, follow up time and age at onset, there was a 14-fold difference in the rates of visual loss (table 8). Young patients with acute anterior uveitis and a short follow up had a visual loss rate of $5 \%$, whereas patients with late onset uveitis of types $2-5$ with a prolonged follow up had a visual loss rate of $69 \%$. Figure 2 shows that the frequency of visual loss does not consistently increase until the age of onset is more than 60 years and the frequency of

\begin{tabular}{|c|c|c|c|c|}
\hline Uveitis type & Total & Idiopathic & Group 2 & Group 3 \\
\hline Acute anterior & 344 & $184(53 \%)$ & $155(45 \%)$ & $5(1 \%)$ \\
\hline Chronic anterior (iris) & 27 & $21(78 \%)$ & $3(11 \%)$ & $3(11 \%)$ \\
\hline Chronic anterior & 45 & $28(62 \%)$ & $7(16 \%)$ & $10(22 \%)$ \\
\hline Choroiditis & 44 & $38(86 \%)$ & $4(9 \%)$ & $2(5 \%)$ \\
\hline Posterior uveitis & 101 & $54(53 \%)$ & $21(21 \%)$ & $26(26 \%)$ \\
\hline
\end{tabular}

Group 2 = sponyloarthropathy, inflammatory bowel disease, psoriasis and diabetes; group 3 = sarcoidosis, multiple sclerosis and Behçet's disease.

Table 3 Complications of uveitis by type of uveitis

\begin{tabular}{lllllll}
\hline Uveitis type & Total & Ocular hypertension & Trabeculectomy & Cataract surgery & All surgery & Systemic treatment \\
\hline Acute anterior & 344 & $24(7 \%)$ & $2(0.6 \%)$ & $17(5 \%)$ & $28(8 \%)$ \\
Chronic anterior (iris) & 27 & $15(56 \%)$ & $4(15 \%)$ & $14(52 \%)$ & $14(52 \%)$ & $5(19 \%)$ \\
Chronic anterior & 45 & $17(38 \%)$ & 0 & $12(27 \%)$ & $15(33 \%)$ & $1(2 \%)$ \\
Choroiditis & 44 & $8(18 \%)$ & $1(2 \%)$ & $3(7 \%)$ & $10(23 \%)$ & $15(34 \%)$ \\
Posterior uveitis & 101 & $24(24 \%)$ & $6(6 \%)$ & $21(21 \%)$ & $22(22 \%)$ \\
\hline
\end{tabular}

Table 4 Level of visual acuity at time of final assessment by type of uveitis

\begin{tabular}{|c|c|c|c|c|c|c|}
\hline Uveitis type & Total & Normal acuity & Unilateral mild & Unilateral severe & Bilateral mild & Bilateral severe \\
\hline Acute anterior & 344 & $313(91 \%)$ & $15(4 \%)$ & $9(3 \%)$ & $7(2 \%)$ & 0 \\
\hline Chronic anterior (iris) & 27 & $14(52 \%)$ & $10(37 \%)$ & $3(11 \%)$ & 0 & 0 \\
\hline Chronic anterior & 45 & $28(62 \%)$ & $9(20 \%)$ & $4(9 \%)$ & $3(7 \%)$ & $1(2 \%)$ \\
\hline Choroiditis & 44 & $25(57 \%)$ & $10(23 \%)$ & $4(9 \%)$ & $5(11 \%)$ & 0 \\
\hline Posterior uveitis & 101 & $71(70 \%)$ & $10(10 \%)$ & $12(12 \%)$ & $5(5 \%)$ & $3(3 \%)$ \\
\hline
\end{tabular}


Table 5 Associations with age of onset of uveitis: univariate analysis

\begin{tabular}{lllll}
\hline & \multicolumn{2}{l}{ No (\%) } & & \\
\cline { 2 - 3 } & Onset $<\mathbf{6 0}$ & Onset $>\mathbf{5 9}$ & Odds ratio (95\% Cl) & Probability \\
\hline Male & $245(52)$ & $35(40)$ & $0.61(0.39$ to 0.98$)$ & 0.039 \\
Follow up $>4$ years & $229(48)$ & $28(32)$ & $0.50(0.31$ to 0.81$)$ & 0.005 \\
Ocular hypertension & $65(14)$ & $23(26)$ & $2.22(1.29$ to 3.82$)$ & 0.004 \\
Cataract & $49(10)$ & $18(20)$ & $2.23(1.23$ to 4.04$)$ & 0.009 \\
Any surgery & $65(14)$ & $24(27)$ & $2.35(1.38$ to 4.03$)$ & 0.002 \\
Visual loss & $78(16)$ & $34(39)$ & $3.19(1.95$ to 5.22$)$ & 0.001 \\
\hline
\end{tabular}

Table 6 Associations with visual loss (univariate analysis)

\begin{tabular}{lllll}
\hline & With visual loss & Without visual loss & p Value & Odds ratio (95\% Cl) \\
\hline Non-white & $11(10 \%)$ & $25(5 \%)$ & 0.048 & $2.1(1.0$ to 4.4$)$ \\
Hospital & $42(38 \%)$ & $238(53 \%)$ & 0.003 & $0.53(0.35$ to 0.81$)$ \\
Acute anterior & $31(28 \%)$ & $315(70 \%)$ & 0.001 & $0.16(0.10$ to 0.26$)$ \\
Systemic disease & & & & \\
$\quad$ None & $60(54 \%)$ & $265(59 \%)$ & & \\
$\quad$ Group & $36(32 \%)$ & $154(34 \%)$ & & \\
$\quad$ Group 2 & $16(14 \%)$ & $30(7 \%)$ & 0.032 & $3.2(2.0$ to 5.2$)$ \\
Age at onset $>60$ years & $34(30 \%)$ & $78(17 \%)$ & 0.001 & $1.9(1.3$ to 2.9$)$ \\
Follow up $>4$ years & $66(59 \%)$ & $191(43 \%)$ & 0.002 & $7.4(4.3$ to 12.8$)$ \\
Cataract & $38(34 \%)$ & $29(6 \%)$ & 0.001 & $4.1(2.5$ to 6.7$)$ \\
Ocular hypertension & $38(34 \%)$ & $50(11 \%)$ & 0.001 & $3.6(1.2$ to 10.4$)$ \\
Trabeculectomy & $6(5 \%)$ & $7(2 \%)$ & 0.017 & $6.2(3.8$ to 10.1$)$ \\
Any surgery & $45(40 \%)$ & $44(10 \%)$ & 0.001 & $3.6(2.2$ to 5.9$)$ \\
Systemic treatment & $34(30 \%)$ & $49(11 \%)$ & 0.001 & \\
Total & 111 & 451 & & \\
\hline
\end{tabular}

Table 7 Multivariate analysis of risk factors for visual loss in uveitis patients and subgroup analysis for acute anterior uveitis and other forms of uveitis

\begin{tabular}{|c|c|c|c|}
\hline & $\begin{array}{l}\text { All patients } \\
\text { OR }(95 \% \mathrm{Cl})\end{array}$ & $\begin{array}{l}\text { Acute anterior } \\
\text { OR }(95 \% \mathrm{Cl})\end{array}$ & $\begin{array}{l}\text { Other uveitis } \\
\text { OR }(95 \% \mathrm{Cl})\end{array}$ \\
\hline $\begin{array}{l}\text { Non-white } \\
\text { Probability }\end{array}$ & & & $\begin{array}{l}4.0(1.1 \text { to } 13.7) \\
0.03\end{array}$ \\
\hline Age onset $>60$ years & $3.9(2.2$ to 7.0$)$ & $3.4(1.4$ to 8.4$)$ & $5.6(2.4$ to 13.1$)$ \\
\hline Probability & 0.001 & 0.008 & 0.001 \\
\hline Acute anterior uveitis & $0.2(0.1$ to 0.3$)$ & & \\
\hline Probability & 0.001 & & \\
\hline Follow up $>4$ years & 2.0 (1.2 to 3.3$)$ & & 2.2 (1.2 to 4.3$)$ \\
\hline Probability & 0.007 & & 0.018 \\
\hline Cataract surgery & $3.9(2.1$ to 7.2$)$ & 6.2 (1.9 to 20.2 ) & 3.5 (1.7 to 7.2$)$ \\
\hline Probability & 0.001 & 0.002 & 0.001 \\
\hline Systemic treatment & & $6.0(2.2$ to 15.9$)$ & $2.6(1.3$ to 5.5$)$ \\
\hline Probability & & 0.001 & 0.01 \\
\hline Hospital & $0.4(0.2$ to 0.6$)$ & & $0.4(0.2$ to 0.7$)$ \\
\hline Probability & 0.001 & & 0.003 \\
\hline Log likelihood & 280 & 104 & 142 \\
\hline Probability & 0.001 & 0.001 & 0.001 \\
\hline
\end{tabular}

Table 8 Frequency of visual loss in uveitis patients grouped by age at onset, type of uveitis, and length of follow up

\begin{tabular}{llll}
\hline & & \multicolumn{2}{l}{ Follow up } \\
\cline { 3 - 4 } Uveitis type & Age (years) at onset & $<\mathbf{4}$ years & $>\mathbf{4}$ years \\
\hline Acute anterior & $<60$ & $8 / 164(5 \%)$ & $13 / 135(10 \%)$ \\
\multirow{2}{*}{ Others } & $>60$ & $7 / 35(20 \%)$ & $3 / 12(25 \%)$ \\
& $<60$ & $18 / 80(23 \%)$ & $39 / 94(41 \%)$ \\
& $>60$ & $13 / 25(52 \%)$ & $11 / 16(69 \%)$ \\
\hline
\end{tabular}




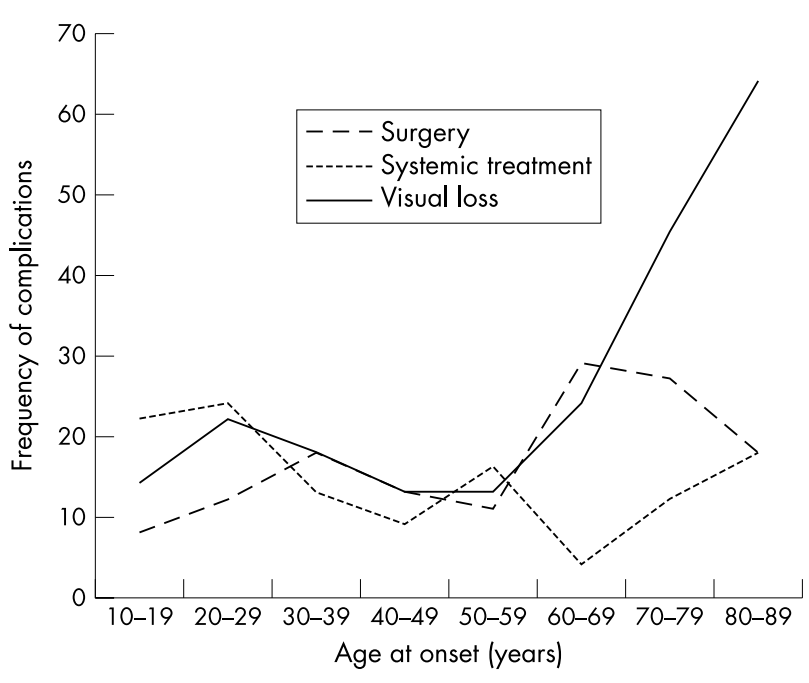

Figure 2 Frequency of complications in patients with uveitis at time of final assessment by age at onset.

surgery increases at a similar age. In contrast, the frequency of systemic treatment shows no increase with age.

In all age groups, apart from the youngest, mild unilateral visual loss was the most frequent category of visual loss (data not shown). The largest numbers of patients with visual loss were those whose uveitis began between 30 and 49 years of age which was also the age at which uveitis was most prevalent (fig 1). In contrast, there were similar numbers of patients with bilateral visual loss in all age groups.

The frequency of visual loss was also analysed by the age at the time of final assessment, as opposed to the age at onset of uveitis, in order that comparison could be made with recent population based studies examining the prevalence of visual loss (fig 3). Again the increased frequency of visual loss is only apparent after the age of 60 years.

\section{DISCUSSION}

This paper represents the first large survey of the characteristics of uveitis presenting to primary referral centres in the United Kingdom. It therefore may reflect the characteristics of uveitis in the general population more accurately than studies based in tertiary referral centres but cannot provide equivalent information to a population based survey. ${ }^{7}$

Idiopathic acute anterior uveitis was the most common type of uveitis seen in our population consistent with other north European studies. ${ }^{15-17}$ The low numbers of non-white people in our study population may have very different risk

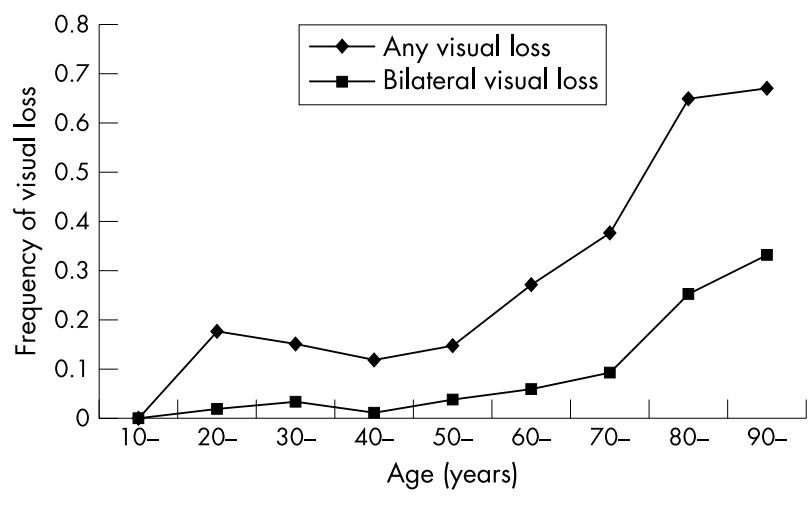

Figure 3 Frequency of visual loss in uveitis patients by age at time of final assessment. Any visual loss $=$ any eye $<6 / 9$; bilateral visual loss = better eye $<6 / 9$. factors for visual loss. There are wide geographic and racial differences in the types of uveitis and the results of this survey may not be relevant in other regions where posterior segment inflammation is more prevalent. ${ }^{18}$ Acute anterior uveitis was associated with the lowest rate of complications in this study, but because of the frequency of this type of uveitis, it contributes significantly to the overall morbidity of uveitis in the general population.

We found that $20 \%$ of our patients had suffered some visual loss. The rate of uniocular visual loss in Darrell et al's hospital based survey of 1962 was similar at 6/34 cases $(18 \%) .{ }^{8}$ Darrell et al also demonstrated that the prevalence of visual loss in acute anterior uveitis $(4 \%)$ was significantly less than that found in posterior segment inflammation (40$43 \%$ ). The similarity of these figures to our own may suggest there have been few effective advances in the management of uveitis in this period but comparison is difficult without details of the hospital referral patterns. Even if there were similar presentation rates to hospital departments, the rate of follow up of burnt out, but visually impaired, cases and the proportion of elderly patients is likely to have great influence on the rates of visual loss seen in cross sectional, hospital based studies.

Rothova et al $^{1}$ found a rate of visual impairment $(<6 / 20)$ of $35 \%$ in 582 Dutch patients with inflammatory eye disease from referral centres. They excluded disease successfully treated in the community, increasing the likelihood of increased severity and prolonged disease in their survey. The most striking difference in their study from our own is the frequency of unilateral or bilateral severe visual loss $(<6 / 60)$. In uveitis patients they found a frequency of 101/560 (18\%) compared to $36 / 561(6.4 \%)$ in our study. There are similarities between the two studies: both studies found that three quarters of the patients with visual loss only had it in one eye; both found that the presence or type of systemic inflammatory disease had little association with the risk of visual loss. The rarity of legal blindness in both studies suggests that national registers of the visually impaired are only going to provide limited information about the contemporary visual impact of uveitis.

In a more recent study Bodaghi et al ${ }^{19}$ found similar rates of severe visual impairment $(<6 / 60)$ in $3.1 \%$ of bilateral and $9.7 \%$ of unilateral cases after treatment; this study was confined to cases of severe uveitis seen at a referral centre.

Rothova et al indicated the importance of follow up in assessing risk factors for visual loss: the rate of surgery in their study increased from $7 \%$ in those followed up for 1 year or less, compared to $41 \%$ in those followed up for more than 3 years. The independent association of surgery and length of follow up with visual loss that we have found suggests that the length of persistent inflammation is likely to have an independent effect on outcome. Only long term prospective studies can provide the most accurate estimates of lifetime visual loss in uveitis, and longitudinal studies of sarcoid, ${ }^{20}$ birdshot retinochoroidopathy, ${ }^{21}$ and juvenile idiopathic arthritis $^{22}$ suggest that follow up needs to be at least 10 years before the lifelong visual morbidity of chronic uveitis can be assessed.

All populations have increasing visual loss with age and many of the complications of uveitis are identical to those found in the normal, elderly population. In this study we specifically investigated the impact of age of onset on the visual burden of uveitis. Two previous studies have found conflicting associations between visual loss and age at onset: age over 40 at onset was found to be associated with a worse visual prognosis in sarcoid ${ }^{20}$ but childhood onset pars planitis is reported to have a worse outcome than that starting in adulthood. ${ }^{23}$ 
Our recent study of paediatric uveitis found a similar frequency of visual loss of $17 \%$ and also found uveitis types other than acute anterior, surgery, and systemic treatment to be significant risk factors. ${ }^{3}$ In contrast, there was no association with age at onset of uveitis in this group of patients despite the likelihood of late presentation being more frequent in younger patients.

The causes of an increased rate of visual loss in late onset uveitis, even when controlled for type of uveitis, follow up time, and treatment variables may be for several reasons. The main causes of irreversible visual loss in both the elderly and the uveitis population are due to maculopathy and glaucoma. The comparison we have made with the published rates of visual loss found in the normal population suggest that uveitis patients do have an increased prevalence of visual loss, of about $5 \%$, from the sixth decade onwards. The rates of bilateral visual loss in the younger uveitis patients are too small to make a meaningful comparison.

The additional burden of uveitis in the elderly may be because of the decreased ability of the ageing eye to tolerate inflammation or because of the additive effect of uveitis with the common co-morbidity of elderly populations. ${ }^{24}$ A third possibility is the increasing risk of delays in presentation and diagnosis that may occur in an elderly population that have coexisting pathology. Late presentation is a major cause of poor outcome in patients with uveitis and both the elderly and young children are more likely to do this. Increasing recognition of the severe impact that uveitis has in the elderly may help more timely diagnosis and treatment of this group of patients. This study demonstrates that in an increasingly ageing population it is important to provide accurate age matched controls if an accurate assessment of the impact of a single disease with multiple, shared complications is to be made. A prospective study with age matched normal controls is the only method to confirm the independent effect of age of onset on the outcome of uveitis and its complications.

We have shown that some groups of uveitis patients still have considerable visual loss despite recent advances in understanding and treatment of this disease. Blindness may now be uncommon but lesser levels of visual loss are little different from previous studies. In order to reduce preventable visual loss, the most important patient subgroup for specialised care would appear to be those patients with painless uveitis shortly after the onset of disease. Reduction of visual loss in those requiring surgery would probably have the greatest overall impact in reducing the visual morbidity in the uveitis population. Whether early referral of high risk patients to units with both medical and surgical experience in uveitis might be the most effective method of reducing the overall rate of visual loss requires further study.

\section{Authors' affiliations}

R Maini, J O'Sullivan, A Reddy, S Watson, C Edelsten, Ipswich Hospital, Suffolk, UK

J O'Sullivan, Frimley Park Hospital NHS Trust, Frimley, Surrey, UK

S Watson, C Edelsten, Prince Charles Eye Unit, Windsor, UK

\section{REFERENCES}

1 Rothova A, Suttorp-van Schulten MSA, Treffers WF, et al. Causes and frequency of blindness in patients with intraocular inflammatory disease. $\mathrm{Br} J$ Ophthalmol 1996:80:332-6.

2 Chatzistefanou K, Markomichelakis NN, Christen W, et al. Characteristics of uveitis presenting for the first time in the elderly. Ophthalmology 1998; 105:347-52.

3 Edelsten C, Reddy MA, Stanford MR, et al. Visual loss associated with pediatric uveitis in English primary and referral centres. Am J Ophthalmol 2003;5:676-80

4 Barton K, Pavesio CE, Towler HM, et al. Uveitis presenting de novo in the elderly. Eye 1994;8:288-91.

5 Munoz B, West SK, Rubin GS, et al. Causes of blindness and visual impairment in a population of older Americans. Arch Ophthalmol 2000;118:819-25

6 Weih LM, Mylan RV, McCarty CA, et al. Age-specific causes of bilateral visual impairment. Arch Ophthalmol 2000;118:264-9.

7 Dandona L, Dandona R, John RK, et al. Population based assessment of uveitis in an urban population in southern India. Br J Ophthalmol 2000;84:706-9.

8 Darrell RW, Wagener HP, Kurland LT, et al. Epidemiology of uveitis. Arch Ophthalmol 1962;68:502-14.

9 Bloch-Michel E, Nussenblatt RB. International Uveitis Study Group recommendations for the evaluation of intraocular inflammatory disease. Am J Ophthalmol 1987; 103:234-5.

10 McCannel CA, Holland GN, Helm CJ, et al. Causes of uveitis in the general practice of ophthalmology. Am J Ophthalmol 1996;121:35-46.

11 La Hey E, Baarsma GS, De Vries J, et al. Clinical analysis of Fuchs' heterochromic cyclitis. Doc Ophthalmol 1991;78:225-35.

12 Leisegang T. Clinical features and prognosis in Fuch's uveitis syndrome. Arch Ophthalmol 1982;100:1622-6.

13 Falcon MG, Williams HP. Herpes simplex keratouveitis and glaucoma. Trans Ophthalmol Soc UK 1978;86:101-4.

14 Van der Lelij A, Ooijman FM, Kijlstra A, et al. Anterior uveitis with sectorial iris atrophy in the absence of keratitis. Ophthalmology 2000;107:1 164-70.

15 Saouli N, Brezin AP. Ocular hypertension and uveitis. Study of 374 cases of uveitis. J Fr Ophtalmol 1999:22:943-9.

16 Saari KM, Paivonsalo-Hietanen T, Vaahtoranta-Lehtonen $\mathrm{H}$, et al. Epidemiology of endogenous uveitis in southwestern Finland. Acta Ophthalmol Scand 1995:345-9.

17 Tran VT, Auer C, Guex-Crosier Y, et al. Epidemiology of uveitis in Switzerland. Ocul Immunol Inflammation 1994:2:169-76.

18 Merrill PT, Kim J, Cox TA, et al. Uveitis in the southeastern United States. Curr Eye Res 1997; 16:865-74.

19 Bodaghi B, Cassoux N, Wechsler B, et al. Chronic severe uveitis. Etiology and visual outcome in 927 patients from a single center. Medicine $2001 ; 80: 263-70$.

20 Edelsten C, Pearson A, Joynes E, et al. The ocular and systemic prognosis of patients presenting with sarcoid uveitis. Eye 1999;13:748-53.

21 Oh KT, Christmas NJ, Folk JC. Birdshot retinochoroiditis: long term follow-up of a chronically progressive disease. Am J Ophthalmol 2002;133:622-9.

22 Edelsten C, Lee V, Bentley CR, et al. An evaluation of baseline risk factors predicting severity in juvenile idiopathic arthritis associated uveitis and other chronic anterior uveitis in early childhood. Br J Ophthalmol 2002;86:51-6.

23 Guest S, Funkhouser E, Lightman S. Pars planitis: a comparison of childhood onset and adult onset disease. Clin Exp Ophthalmol 2001;29:81-4.

24 Wakefield D, McCluskey PJ, Dunlop I, et al. Uveitis: aetiology and disease associations in an Australian population. Aust N Z J Ophthalmol

1986;14:181-7. 